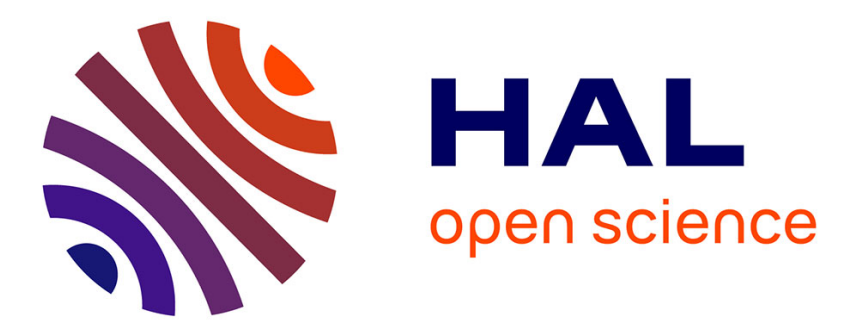

\title{
L'analyse de la géographie des flux touristiques en France métropolitaine: un autre regard sur l'attractivité des territoires \\ Patricia Lejoux
}

\section{- To cite this version: \\ Patricia Lejoux. L'analyse de la géographie des flux touristiques en France métropolitaine: un autre regard sur l'attractivité des territoires. Flux - Cahiers scientifiques internationaux Réseaux et terri- toires, 2006. halshs-02491509}

\section{HAL Id: halshs-02491509 \\ https://shs.hal.science/halshs-02491509}

Submitted on 26 Feb 2020

HAL is a multi-disciplinary open access archive for the deposit and dissemination of scientific research documents, whether they are published or not. The documents may come from teaching and research institutions in France or abroad, or from public or private research centers.
L'archive ouverte pluridisciplinaire HAL, est destinée au dépôt et à la diffusion de documents scientifiques de niveau recherche, publiés ou non, émanant des établissements d'enseignement et de recherche français ou étrangers, des laboratoires publics ou privés. 


\title{
L'ANALYSE DE LA GÉOGRAPHIE DES FLUX TOURISTIQUES EN FRANCE MÉTROPOLITAINE : UN AUTRE REGARD SUR L'ATTRACTIVITÉ DES TERRITOIRES
}

\author{
Patricia Lejoux \\ Métropolis | Flux
}

$2006 / 3-n^{\circ} 63$

pages 33 à 46

ISSN 1154-2721

Article disponible en ligne à l'adresse:

http://www.cairn.info/revue-flux-2006-3-page-33.htm

Pour citer cet article :

Lejoux Patricia, « L'analyse de la géographie des flux touristiques en France métropolitaine : un autre regard sur l'attractivité des territoires »,

Flux, 2006/3 n63, p. 33-46.

Distribution électronique Cairn.info pour Métropolis.

(C) Métropolis. Tous droits réservés pour tous pays.

La reproduction ou représentation de cet article, notamment par photocopie, n'est autorisée que dans les limites des conditions générales d'utilisation du site ou, le cas échéant, des conditions générales de la licence souscrite par votre établissement. Toute autre reproduction ou représentation, en tout ou partie, sous quelque forme et de quelque manière que ce soit, est interdite sauf accord préalable et écrit de l'éditeur, en dehors des cas prévus par la législation en vigueur en France. II est précisé que son stockage dans une base de données est également interdit. 


\title{
L'analyse de la géographie des flux touristiques en France métropolitaine: un autre regard sur l'attractivité des territoires
}

\author{
Patricia Lejoux
}

\section{LES COMPOSANTES DE l'ATTRACTIVITÉ TERRITORIALE: DE LA LOCALISATION DES ENTREPRISES A LA LOCALISATION DES INDIVIDUS} 'attractivité territoriale apparaît, aujourd'hui, comme un Lthème central des politiques de développement local (1). Le renforcement de la capacité des territoires à attirer les entreprises figure, en effet, dans nombre de projets locaux comme l'objectif essentiel des stratégies de développement et se mesure généralement à travers l'évolution des flux d'investissement internationaux ou le nombre de créations d'établissements. L'apparition de cette thématique au sein des politiques de développement local s'explique principalement par la transformation du contexte économique intervenue depuis le milieu des années 1980 et par la modification des critères d'implantation des entreprises qui s'en est suivie. L'émergence d'un nouvel environnement économique, marqué par la mondialisation des échanges et l'évolution des modes d'organisation de la production, a, en effet, contribué à redéfinir les stratégies de localisation des firmes. Celles-ci semblent, aujourd'hui, déterminées par différents facteurs: les arbitrages entre rendements d'échelle croissants et coûts de transport, les externalités positives associées à la concentration géographique, et les effets de la concurrence spatiale (Gérard-Varet, Mougeot, 2001). Or, cette évolution des critères d'implantation des entreprises semble surtout bénéficier aux espaces les plus développés, au premier rang desquels figurent les métropoles (Thisse, Van Ypersele, 1999). Situés au cœur des réseaux de télécommunications, routiers, ferroviaires et logistiques, les espaces métropolitains apparaissent, ainsi, comme les principaux pôles de croissance autour desquels se développent les flux d'échanges et de production.
L'émergence de cette "économie d'archipel », où les relations entre pôles deviennent prédominantes, contribue aujourd'hui à accentuer les inégalités spatiales, les espaces périphériques restant à l'écart des logiques de développement (Veltz, 1996). Ainsi, dans un nouveau contexte où les choix de localisation des firmes apparaissent plus discriminants pour les espaces, il semble naturel que la capacité des territoires à attirer et retenir des entreprises devienne un enjeu crucial des politiques de développement local.

Cependant, les enjeux de l'attractivité territoriale peuventils se résumer uniquement à la capacité des territoires à attirer les entreprises? Leur propension à attirer les individus ne constitue-t-elle pas également un aspect essentiel de leur attractivité, et par-delà, de leur développement? Si les travaux de la nouvelle économie géographique initiés par Paul Krugman (Krugman, 1991) ont incontestablement renouvelé les approches de la géographie économique en mettant en évidence le phénomène de polarisation croissante des activités, ils ont également contribué à diffuser une vision du développement local centrée essentiellement sur les processus liés à la production. Or, la question du développement local ne se limite pas à la seule problématique de l'offre, mais recouvre également celle de la demande et de la consommation. Si la localisation des entreprises a été modifiée, au cours de ces dernières années, la localisation des individus a connu également d'importants changements sous l'effet de l'accroissement des mobilités et de l'éclatement spatial des espaces de vie. Longtemps associés à un lieu unique, les ménages travaillant, résidant et consommant à proximité, le rapport des individus à l'espace semble marqué, aujourd'hui, par une multiplication des lieux 
de vie à travers la dissociation croissante des zones de travail, de résidence, de loisirs, de tourisme (Knafou, 2000). Or, ce phénomène s'apparente, sur le plan économique, à un processus de dissociation des lieux de production et de consommation dans la vie des ménages et celui-ci semble avoir des incidences importantes sur les économies locales (Davezies, 2004). En effet, les revenus produits sur un territoire n'y sont plus automatiquement consommés, ce qui entraîne des transferts de richesse au profit d'espaces qui semblent particulièrement attractifs sur le plan résidentiel (Davezies, 2003). Ces éléments mettent ainsi en évidence l'importance que peut recouvrer pour un territoire la présence de populations qui viennent consommer localement. La question du développement local ne semble donc pas être déterminée seulement par l'attractivité des territoires vis-à-vis des entreprises, même si celle-ci reste bien entendu essentielle, mais également par leur attractivité vis-à-vis des individus qui sont aussi des consommateurs.

Les mobilités touristiques, qui correspondent à des déplacements d'au moins une nuitée effectués hors de l'environnement habituel, jouent un rôle central dans ce processus. Leur accroissement considérable, favorisé par l'augmentation du temps libre et le développement des réseaux de transport, a, en effet, contribué à complexifier la localisation spatiale des individus et à accentuer ce processus de dissociation des lieux de production et de consommation dans la vie des ménages. Les mobilités touristiques amènent, ainsi, à repenser les modes d'occupation de l'espace, car les individus ne sont plus en permanence sur leur lieu de résidence principale, ce qui introduit un décalage entre la vision traditionnelle de la population résidente et la réalité de la population présente (Terrier et alii, 2005). Ce phénomène tend, d'ailleurs, à s'intensifier avec l'émergence de nouvelles pratiques spatiales qui transcendent le cadre strict du tourisme comme les déplacements à longue distance effectués dans la journée ou les pratiques de double résidence (Bonnin \& Villanova, 1999; Dubost, 1998). Or, ces mobilités touristiques s'apparentent à un déplacement de consommation du lieu de résidence vers le lieu de séjour, ce qui explique que les liens entre les espaces où les revenus sont générés et ceux où ils sont dépensés se distendent (Claval, 2003). Ces éléments soulignent l'importance que pourrait recouvrer pour les territoires la présence de touristes qui viennent consommer localement. Surtout, lorsqu'on sait que la consommation touristique en France s'élève à plus de 100 milliards d'euros et qu'elle représente près de $6,6 \%$ du produit intérieur brut. Ainsi, la capacité des territoires à attirer temporairement des populations, au premier rang desquelles figurent les touristes et les résidents secondaires, semble constituer une autre composante importante de l'attractivité territoriale et du développement local. D'autant plus que ces mobilités temporaires peuvent parfois se révéler prescriptives de mobilités définitives, à travers les migrations de retraite, voire les migrations d'actifs, qui semblent aujourd'hui s'orienter principalement vers les régions bénéficiant d'un cadre de vie agréable (Baccaïni, 2001).

Les mobilités touristiques semblent constituer, ainsi, des marqueurs de l'attractivité globale des espaces vis-à-vis des individus et la connaissance de leur géographie pourrait, alors, contribuer à apporter un autre regard sur cette thématique de l'attractivité territoriale et sur ses enjeux en matière de développement local. Mais, que sait-on, aujourd'hui, précisément de la géographie des mobilités touristiques? Quels sont les espaces attractifs vis-à-vis des touristes? Pour répondre à ces questions, nous proposons d'analyser la structure des flux touristiques en France métropolitaine et d'étudier la géographie de l'attractivité des espaces français à l'égard des flux touristiques.

\section{L'ÉTUDE DES FLUX TOURISTIQUES: NIVEAU D'ANALYSE ET APPROCHE MÉTHODOLOGIQUE}

Il convient, tout d'abord, de préciser que les flux touristiques sont beaucoup plus souvent étudiés au niveau international qu'au niveau national. En effet, la plupart des ouvrages sur le tourisme (2) traitent essentiellement de la répartition spatiale des flux touristiques au niveau mondial, c'est-à-dire des déplacements entre pays ou entre ensembles régionaux (Europe, Asie-Pacifique, Amériques...). Ceux-ci ont connu un accroissement considérable et constituent, aujourd'hui, un aspect essentiel de la mondialisation: on dénombrait, ainsi, 25 millions d'arrivées de touristes internationaux en 1950, 612 millions en 1997 et d'après I'OMT (Organisation Mondiale du Tourisme) elles s'élevaient à 760 millions en 2004. La structure de ces flux touristiques internationaux est relativement bien connue, puisque les données publiées par l'OMT permettent d'identifier les principaux foyers émetteurs et récepteurs du tourisme mondial. Cette approche met, ainsi, en évidence le rôle de la France dans l'organisation spatiale des flux touristiques internationaux: celle-ci constitue un pôle incontournable, puisqu'elle représente la première destination touristique mondiale avec plus de 75 millions d'arrivées de touristes internationaux par an, ce qui 
Les déplacements touristiques des Français sont mesurés ici à partir des données de l'enquête Suivi de la demande touristique française (SDT), réalisée par la Direction du tourisme en partenariat avec la SOFRES. Basée sur l'interrogation mensuelle d'un panel de 20000 individus (10000 jusqu'en mars 1999) représentatifs de la population française âgée de 15 ans et plus, elle permet de connaître les déplacements touristiques des Français en France et à l'étranger sur une année et notamment leur région d'origine et de destination.

La taille de l'échantillon apparaissant limitée pour construire une matrice des flux interrégionaux sur une année, les résultats font appel ici à des moyennes établies sur la période 1997-2001 à partir du volume de nuitées extrapolées.

Les déplacements touristiques des étrangers, faute d'une Enquête aux frontières depuis 1996, sont mesurés ici à partir des estimations annuelles réalisées par la Direction du tourisme, sur la même période. Celle-ci a développé en effet une méthodologie spécifique basée sur l'estimation des dépenses mensuelles des étrangers en France puis de l'estimation des volumes annuels d'arrivées et de nuitées, notamment au niveau régional.

équivaut à $10 \%$ des arrivées mondiales. Ainsi, l'attrait exercé par la France vis-à-vis des touristes étrangers semble constituer une caractéristique essentielle du tourisme en France: en effet, malgré l'émergence de nouvelles destinations, celle-ci a réussi à maintenir son rang de premier pays récepteur de flux touristiques internationaux sur les dix dernières années (3). Parallèlement, elle fait également partie des grands pays émetteurs avec ses voisins européens (Royaume-Uni, Allemagne, Pays-Bas...), bien qu'à la différence de ceux-ci, les déplacements vers l'étranger restent une pratique minoritaire chez les touristes français.

Si l'étude de la structure des flux touristiques au niveau mondial contribue donc à mettre en évidence l'attractivité de la France vis-à-vis des touristes étrangers, elle tend, par contre, à faire oublier une autre particularité du tourisme hexagonal: l'attractivité touristique exercée par le pays sur ses propres habitants. En effet, les déplacements touristiques des Français se font majoritairement en France: près de $85 \%$ des nuitées des touristes français sont effectuées dans l'hexagone. Mais, à l'exception de quelques travaux (4), peu d'études s'intéressent à l'analyse de l'origine et de la destination de ces déplacements, et à la manière dont ils se combinent avec les déplacements touristiques des étrangers. Or, dans un contexte où l'attractivité des espaces vis-à-vis des populations non-résidentes pourrait avoir des incidences en matière de développement local, il semble particulièrement intéressant de connaître la structure géographique des flux touristiques au niveau national. En fait, seules les publications de la Direction du Tourisme et de l'Observatoire National du Tourisme apportent des éléments de connaissance sur l'origine et la destination des déplacements touristiques en France (5). Il est possible de connaître la répartition des nuitées touristiques effectuées en France par région réceptrice ou émettrice et d'identifier, ainsi, les grandes régions touristiques. Mais ce classement, établi en fonction du volume de nuitées touristiques effectuées dans chaque région, est souvent assimilé à une estimation de leur attractivité touristique. Les régions ayant accueilli le volume le plus important de nuitées touristiques étant, alors, considérées comme les plus attractives. Or, ces éléments renseignent davantage sur le classement des principales destinations touristiques et leurs parts de marché respectives, que sur l'attractivité réelle des régions. Les déplacements touristiques sont, en effet, le produit d'une interaction spatiale entre des lieux d'origine et de destination, et leur analyse nécessite donc de considérer chaque espace dans sa double fonction d'émission et de réception des nuitées touristiques. L'attractivité touristique des régions doit donc être évaluée en tenant compte à la fois du volume des nuitées touristiques effectuées dans la région, mais également du volume des nuitées touristiques émises par les habitants de la région à l'extérieur de celle-ci.

Cette approche sera développée ici pour étudier l'attractivité des régions françaises à l'égard des touristes français et étrangers. Nous nous proposons d'effectuer une analyse détaillée de la structure des flux touristiques en France métropolitaine, au niveau régional, en considérant chaque région dans sa double fonction d'émission et de réception. Nous nous intéresserons, dans un premier temps, aux flux touristiques internes, c'est-àdire aux déplacements touristiques des Français en France métropolitaine, et nous intégrerons ensuite les flux touristiques internationaux, c'est-à-dire les déplacements touristiques en provenance et à destination de l'étranger. Ces éléments devraient nous permettre d'évaluer l'attractivité globale des régions vis-à-vis des touristes français et étrangers, et de mettre en évidence les enjeux différenciés que représente la gestion des flux touristiques pour les régions françaises. 


\section{LeS FLUX TOURISTIQUeS DES FranÇAIS EN FrANCE MÉTROPOLITAINE}

Avant d'étudier plus spécifiquement l'attractivité touristique des régions, il semble nécessaire de présenter, d'abord, les principales caractéristiques de la structure des flux touristiques des Français en France métropolitaine. Il s'agit donc ici d'identifier les principales régions émettrices et réceptrices, et d'analyser l'origine et la destination de ces flux touristiques.

\section{Des régions inégalement et très différemment investies par les flux touristiques}

Les déplacements touristiques des Français en France métropolitaine se caractérisent, tout d'abord, par l'éloignement des lieux de séjours par rapport aux lieux de résidence. En effet, les déplacements de proximité sont rares, puisque près de $84 \%$ des nuitées touristiques sont effectuées dans une autre région que la région de résidence. Ainsi, les mobilités touristiques s'apparentent bien à un phénomène de dissociation des lieux de production et de consommation dans la vie des ménages, les espaces de travail et de résidence se différenciant clairement des espaces de séjours et de vacances. Cependant les situations sont très différentes d'une région à l'autre, car celles-ci sont très inégalement impliquées dans l'émission et la réception des flux touristiques. Il existe, en effet, une forte concentration spatiale des flux touristiques, puisque quatre régions émettent à elles seules plus de la moitié des flux touristiques (Ile-de-France, Rhône-Alpes, Provence-Alpes-Côte d'Azur, Nord-Pas-de-Calais) et, parallèlement, six régions en captent plus de la moitié (PACA, Rhône-Alpes, Languedoc-Roussillon, Bretagne, Aquitaine, Pays de la Loire).

Si, dans la plupart des espaces français, les flux émis et reçus s'équilibrent à peu près, certaines régions au contraire se caractérisent par leur fonction émettrice ou réceptrice. Ainsi, les régions du Nord et de l'Est sont des régions essentiellement émettrices: on y retrouve l'lle-de-France, le Nord-Pas-de-Calais, la Haute-Normandie, I'Alsace, la Lorraine et la ChampagneArdenne. L'Ile-de-France occupe une place privilégiée au sein de cet ensemble, puisqu'elle arrive largement en tête des régions émettrices, en terme de nuitées par habitant. Elle génère, d'ailleurs, à elle seule, près de $30 \%$ des nuitées touristiques françaises effectuées en France métropolitaine. Cette situation s'explique aisément par la surmobilité touristique des Franciliens: leur taux de départ est supérieur de près de 13 points à la moyenne nationale et le nombre moyen de voyages par individu est de 5,4 en Île-de-France, contre 4,2 en France métropolitaine. La région francilienne cumule, en effet, tous les facteurs propices à la mobilité: cadre de vie fortement urbanisé, revenus élevés, proportion de cadres plus importante qu'en province, très bonne qualité des dessertes ferroviaires, autoroutières et aériennes... De leur côté, les régions du Sud et de l'Ouest sont des régions essentiellement réceptrices, la Corse et le Languedoc-Roussillon arrivant largement en tête, suivies des régions PACA, Poitou-Charentes, Bretagne et Aquitaine. Les fortes aménités naturelles de ces espaces en font des destinations privilégiées pour les habitants des autres régions françaises, la mer jouant ici un rôle essentiel, puisque les flux touristiques se font majoritairement à destination des départements littoraux. Mais c'est aussi sur ces côtes que se concentrent les principales capacités d'accueil en hébergements touristiques (hôtels, campings, résidences de tourisme, résidences secondaires...).

Ces premiers éléments mettent en évidence la situation très contrastée des régions françaises à l'égard des flux touristiques des Français en France métropolitaine. En effet, des profils territoriaux très marqués apparaissent, avec des régions essentiellement réceptrices et d'autres essentiellement émettrices. Cette approche par les flux montre, ainsi, que le tourisme ne se traduit pas systématiquement par un gain pour les territoires, par conséquent leur attractivité à l'égard de leurs propres résidents semble un enjeu tout aussi important que leur attractivité vis-àvis de l'extérieur. En particulier, si I'on envisage les déplacements touristiques comme des déplacements de consommation: si dans le cas des régions réceptrices les mobilités touristiques peuvent être assimilées à un "bénéfice ", pour les régions émettrices elles s'apparentent au contraire à une perte de richesse, ce qui pourrait avoir des effets plus ou moins préjudiciables suivant la situation économique des espaces concernés.

Les tendances générales ayant été identifiées, il s'agit maintenant de procéder à un examen plus détaillé de la structure des flux touristiques dans chaque région, en analysant leur origine et leur destination. L'élaboration d'une matrice complète regroupant les 22 régions de France métropolitaine permet d'étudier précisément l'origine et la destination des flux touristiques reçus et émis dans chaque région. Les déplacements intrarégionaux sont donc intégrés cette fois à l'analyse, mais leur prise en compte modifie très peu la structure générale des flux touristiques esquissée précédemment, en particulier, les principales régions réceptrices et émettrices restent les mêmes. 


\begin{tabular}{|c|c|c|}
\hline Régions d'origine & Part des nuitées touristiques effectuées & Principales régions de destination \\
\hline île de France & $29,0 \%$ & $\begin{array}{l}\text { Provence-Alpes-Côte d'Azur } \\
\text { Rhône-Alpes } \\
\text { Bretagne } \\
\text { Pays de la Loire } \\
\text { Languedoc-Roussillon } \\
\text { Aquitaine }\end{array}$ \\
\hline Rhône-Alpes & $9,5 \%$ & $\begin{array}{l}\text { Rhône-Alpes } \\
\text { Provence-Alpes-Côte d'Azur } \\
\text { Languedoc-Roussillon }\end{array}$ \\
\hline Provence-Alpes-Côte d'Azur & $7,0 \%$ & $\begin{array}{l}\text { Provence-Alpes-Côte d'Azur } \\
\text { Rhône-Alpes } \\
\text { Languedoc-Roussillon }\end{array}$ \\
\hline Pays de la Loire & $5,7 \%$ & $\begin{array}{l}\text { Pays de la Loire } \\
\text { Bretagne } \\
\text { Île-de-France } \\
\text { Rhône-Alpes }\end{array}$ \\
\hline Nord-Pas-de-Calais & $5,6 \%$ & $\begin{array}{l}\text { Nord-Pas-de-Calais } \\
\text { Provence-Alpes-Côte d'Azur } \\
\text { Rhône-Alpes } \\
\text { Languedoc-Roussillon }\end{array}$ \\
\hline Régions de destination & Part des nuitées touristiques effectuées & Principales régions d'origine \\
\hline Provence-Alpes-Côte d'Azur & $12,9 \%$ & $\begin{array}{l}\text { Île-de-France } \\
\text { Provence-Alpes-Côte d'Azur } \\
\text { Rhône-Alpes }\end{array}$ \\
\hline Rhône-Alpes & $11,4 \%$ & $\begin{array}{l}\text { Île-de-France } \\
\text { Rhône-Alpes } \\
\text { Provence-Alpes-Côte d'Azur }\end{array}$ \\
\hline Languedoc-Roussillon & $9,2 \%$ & $\begin{array}{l}\text { Île-de-France } \\
\text { Rhône-Alpes } \\
\text { Languedoc-Roussillon } \\
\text { Midi-Pyrénées }\end{array}$ \\
\hline Bretagne & $8,1 \%$ & $\begin{array}{l}\text { Île-de-France } \\
\text { Bretagne } \\
\text { Pays de la Loire }\end{array}$ \\
\hline Aquitaine & $7,8 \%$ & $\begin{array}{l}\text { Île-de-France } \\
\text { Aquitaine } \\
\text { Midi-Pyrénées }\end{array}$ \\
\hline Pays de la Loire & $7,2 \%$ & $\begin{array}{l}\text { Île-de-France } \\
\text { Pays de la Loire }\end{array}$ \\
\hline
\end{tabular}


L'origine des nuitées touristiques effectuées dans chaque région semble marquée par l'importance considérable des flux en provenance de l'lle-de-France. En effet, à l'exception du Nord-Pas-de-Calais, dans chaque région française la part essentielle des nuitées touristiques est réalisée par les Franciliens. Le poids du tourisme francilien est particulièrement élevé dans les régions du Bassin Parisien où l'effet de proximité joue fortement: il représente ainsi plus de $40 \%$ des nuitées touristiques effectuées dans le Centre, en Picardie, en Bourgogne et en Haute-Normandie. Mais il est également important dans les régions très touristiques où il génère plus de $30 \%$ des nuitées, en particulier en Corse et dans les régions de l'Ouest (Bretagne, Pays de la Loire, Poitou-Charentes) qui sont par ailleurs très accessibles depuis I'lle-de-France. Le rôle essentiel du tourisme francilien dans les régions françaises s'explique non seulement par la surmobilité et l'importance de la population francilienne, mais aussi par l'orientation spécifique des flux touristiques en provenance de l'lle-de-France. En effet, contrairement aux autres régions françaises, les flux touristiques des Franciliens ne se concentrent pas sur une ou deux destinations particulières, mais bénéficient au contraire à l'ensemble des régions françaises en se répartissant de manière équilibrée entre elles. L'Ilede-France produisant à elle seule près de $30 \%$ de la richesse nationale, on entrevoit ici le rôle clé que peuvent jouer, pour les espaces français, les déplacements de consommation générés par les mobilités touristiques des Franciliens. La destination des flux touristiques semble, pour sa part, marquée par l'attrait considérable des régions du Sud-est et de l'Ouest. Celles-ci figurent, dans la plupart des régions françaises, au premier ou au second rang des destinations touristiques effectuées par les habitants de chaque région. Le Sud semble particulièrement plébiscité, notamment la région Provence-Alpes-Côte d'Azur comme destination estivale et la région Rhône-Alpes comme destination hivernale. À l'Ouest ce sont vers les régions Bretagne et Pays de la Loire que s'orientent majoritairement les flux touristiques.

L'analyse de l'origine et de la destination des flux touristiques met également en évidence une caractéristique essentielle du tourisme des Français: I'importance du tourisme intrarégional pour certaines régions. Dans 16 régions sur 22, la région de résidence constitue, en effet, la première ou la seconde destination touristique des habitants. Le poids du tourisme intrarégional est naturellement plus élevé dans les régions touristiques du Sud et de l'Ouest, où il représente près de $30 \%$ des nuitées touristiques effectuées par les habitants de la région, notamment en Aquitaine, en Bretagne, et dans les régions Provence-Alpes-Côte d'Azur, Languedoc-Roussillon, Pays de la Loire et Rhône-Alpes. Les fortes aménités naturelles de ces régions incitent, en effet, leurs habitants à rester dans la région pour leurs séjours touristiques. Mais le tourisme intrarégional représente également, dans 17 régions sur 22, le premier ou le second flux le plus important concernant les nuitées touristiques effectuées dans la région. II joue un rôle particulièrement important dans le Nord-Pas-de-Calais et en Rhône-Alpes, où il représente respectivement $36,6 \%$ et $22,9 \%$ des nuitées effectuées dans la région. On retrouve donc ici l'une des spécificités du tourisme français, à savoir le caractère local d'une partie du tourisme national. D'un point de vue économique, l'importance du tourisme intrarégional dans certaines régions constitue un avantage indéniable pour celles-ci, car elles restent ainsi les principales bénéficiaires des déplacements de consommation générés par les déplacements touristiques de leurs habitants.

Les principales caractéristiques de la structure des flux touristiques ayant été identifiées, il s'agit, à présent, d'évaluer l'attractivité des régions à l'égard des touristes français.

\section{L'attractivité différenciée des régions à l'égard des flux touristiques des Français en France métropolitaine}

L'attractivité touristique sera définie ici à la fois par la capacité des régions à attirer des touristes originaires des autres régions françaises, mais aussi par leur capacité à conserver leurs propres habitants comme touristes sur le territoire. Étant donné le rôle essentiel joué par le tourisme intrarégional dans la structure des flux touristiques, il semble indispensable de tenir compte de l'attrait exercé par les régions sur leurs habitants. De plus, c'est bien la conjugaison de ces deux composantes, l'attractivité touristique des espaces vis-à-vis de l'extérieur et vis-àvis de leurs résidents, qui semble aujourd'hui importante en matière de développement local. En effet, les enjeux pour les économies locales étant liés aux consommations générées par la présence de touristes sur le territoire, il apparaît indispensable de prendre en compte aussi bien les nuitées effectuées par les touristes locaux, que par les touristes qui viennent de l'extérieur. L'attractivité touristique des régions sera donc mesurée, ici, par la différence entre le nombre de nuitées effectuées par les touristes dans la région, qu'ils soient originaires ou non de celle-ci, et le nombre de nuitées réalisées par les habitants de la région hors de celle-ci. Afin de pouvoir comparer les régions 


\section{Carte 1 - L'attractivité des régions à l'égard des flux touristiques des Français en France métropolitaine}

Source: calcul de l'auteur, SDT 1997-2001

Direction du tourisme/Sofrès

(C) Arctique

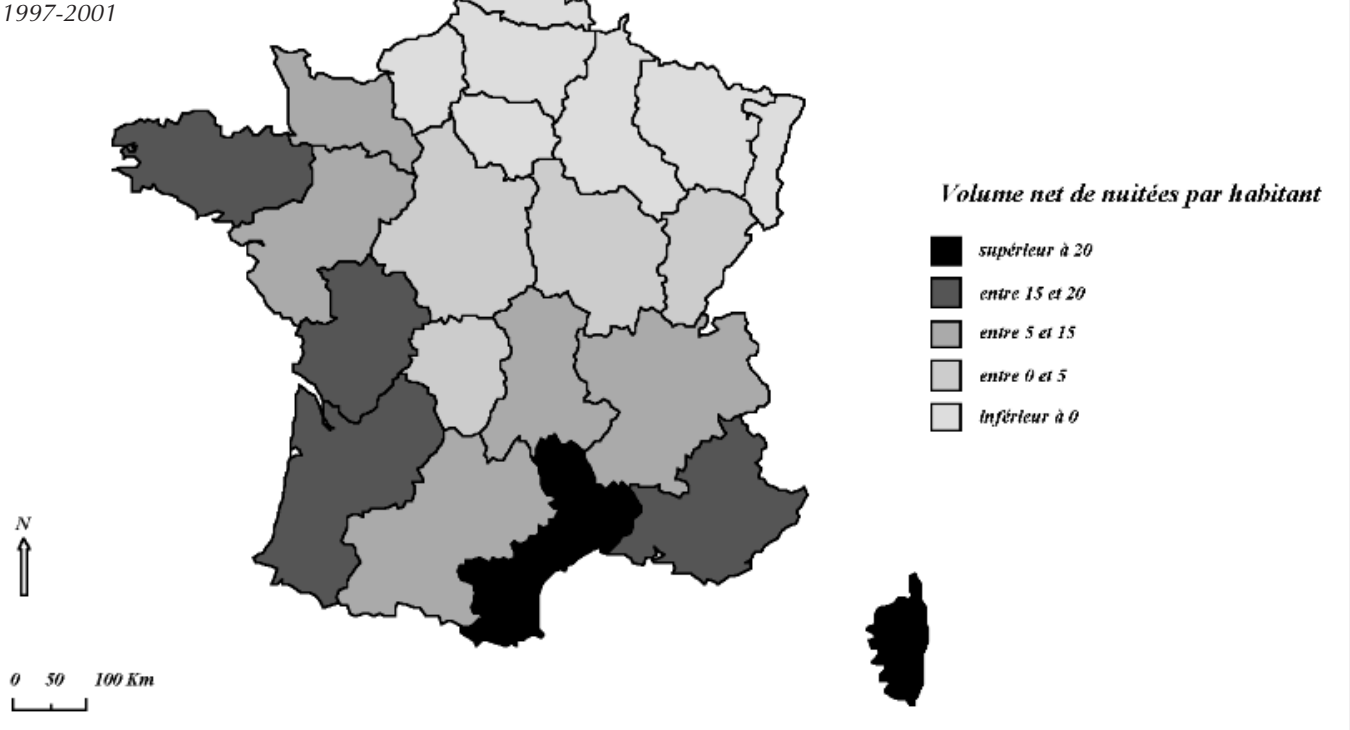

entre elles indépendamment de la taille de leur population, le volume net de nuitées touristiques sera rapporté à la population régionale.

Le fait de prendre en compte non seulement les nuitées effectuées dans la région, mais également les nuitées réalisées à l'extérieur de celle-ci contribue à modifier les représentations traditionnelles de l'attractivité touristique des régions françaises. En effet, celles-ci se fondent d'habitude uniquement sur les nuitées reçues et associent donc systématiquement le tourisme à un gain pour les territoires. Or, l'approche développée ici contribue fortement à relativiser le potentiel attractif de certaines régions et, par conséquent, le bénéfice retiré du tourisme, puisque dans certains cas le volume de nuitées émises à l'extérieur de la région est largement supérieur au volume de nuitées effectuées dans la région. On constate, ainsi, une césure nette entre les régions du Sud et de l'Ouest, qui bénéficient d'un volume net de nuitées par habitant positif, et les régions du Nord et de l'Est, qui disposent d'un volume net de nuitées par habitant négatif. Le climat et l'environnement fortement industrialisé et urbanisé de celles-ci expliquent en partie leur rapport déficitaire. En effet, elles ne semblent pas disposer d'atouts suffisants pour attirer les touristes des autres régions et inciter leurs propres habitants à effectuer leurs séjours touristiques dans leur région de résidence, ce qui se traduit par l'émission importante de flux touristiques vers l'extérieur. Les régions françaises appa- raissent donc très inégalement attractives à l'égard des flux touristiques des Français, la division géographique entre régions attractives et non attractives reproduisant la coupure traditionnelle de la France le long de l'axe Le Havre-Marseille. Ainsi, les régions les moins attractives à l'égard des flux touristiques semblent correspondre aux régions les plus productives, en terme de PIB par emploi et, inversement, les régions les plus attractives coïncident globalement avec les régions les moins productives. L'analyse de l'attractivité des espaces français à l'égard des seuls flux touristiques des Français en France métropolitaine semble, ainsi, esquisser une géographie inverse des espaces de production et de consommation.

Mais le caractère attractif ou non attractif des régions à l'égard des flux touristiques des Français n'a pas la même intensité et ne repose pas sur les mêmes ressorts d'une région à l'autre. Il dépend, en effet, de l'attrait plus ou moins important, qu'elle exerce sur ses habitants et sur les habitants des autres régions françaises. L'analyse détaillée de certaines situations va permettre de mettre en évidence la configuration spécifique des flux touristiques dans chaque région. Nous nous intéresserons, d'abord, à celles qui bénéficient d'un volume net de nuitées par habitant positif, en distinguant les régions très attractives des régions moyennement attractives, puis à celles qui disposent d'un volume net de nuitées par habitant négatif. 
Les ressorts de l'attractivité touristique des régions littorales et centrales: un attrait important vis-à-vis des habitants des autres régions françaises ou de leurs propres habitants

Les régions les plus attractives à l'égard des flux touristiques sont celles qui bordent la Méditerranée et l'Atlantique, avec, en tête, la Corse et le Languedoc-Roussillon, suivies des régions Bretagne, Provence-AlpesCôte d'Azur, Aquitaine et PoitouCharentes. Les régions littorales du Sud et de l'Ouest exercent, en effet, à la fois une très forte attractivité sur les habitants des autres régions françaises et sur leurs propres habitants, ce qui explique leur situation particulièrement avantageuse.

Pour autant, la structure des flux touristiques n'est pas identique d'un espace à l'autre. Ainsi, le LanguedocRoussillon et la Corse se caractérisent surtout par leur très forte attractivité vis-à-vis de l'extérieur. Elles entretiennent, en effet, un rapport particulièrement avantageux avec les autres régions françaises, puisqu'elles reçoivent quatre fois plus de nuitées qu'elles n'en émettent. Elles constituent, d'ailleurs, les deux principales régions réceptrices, en terme de nuitées par habitant. Le LanguedocRoussillon se distingue, toutefois, de la Corse par un pouvoir casanier beaucoup plus important, puisque près de $30 \%$ des nuitées touristiques des habitants du LanguedocRoussillon sont effectuées dans leur
Figure 1 - L'attractivité touristique des régions à l'égard de leurs propres habitants et des habitants des autres régions françaises

Pouvoir casanier

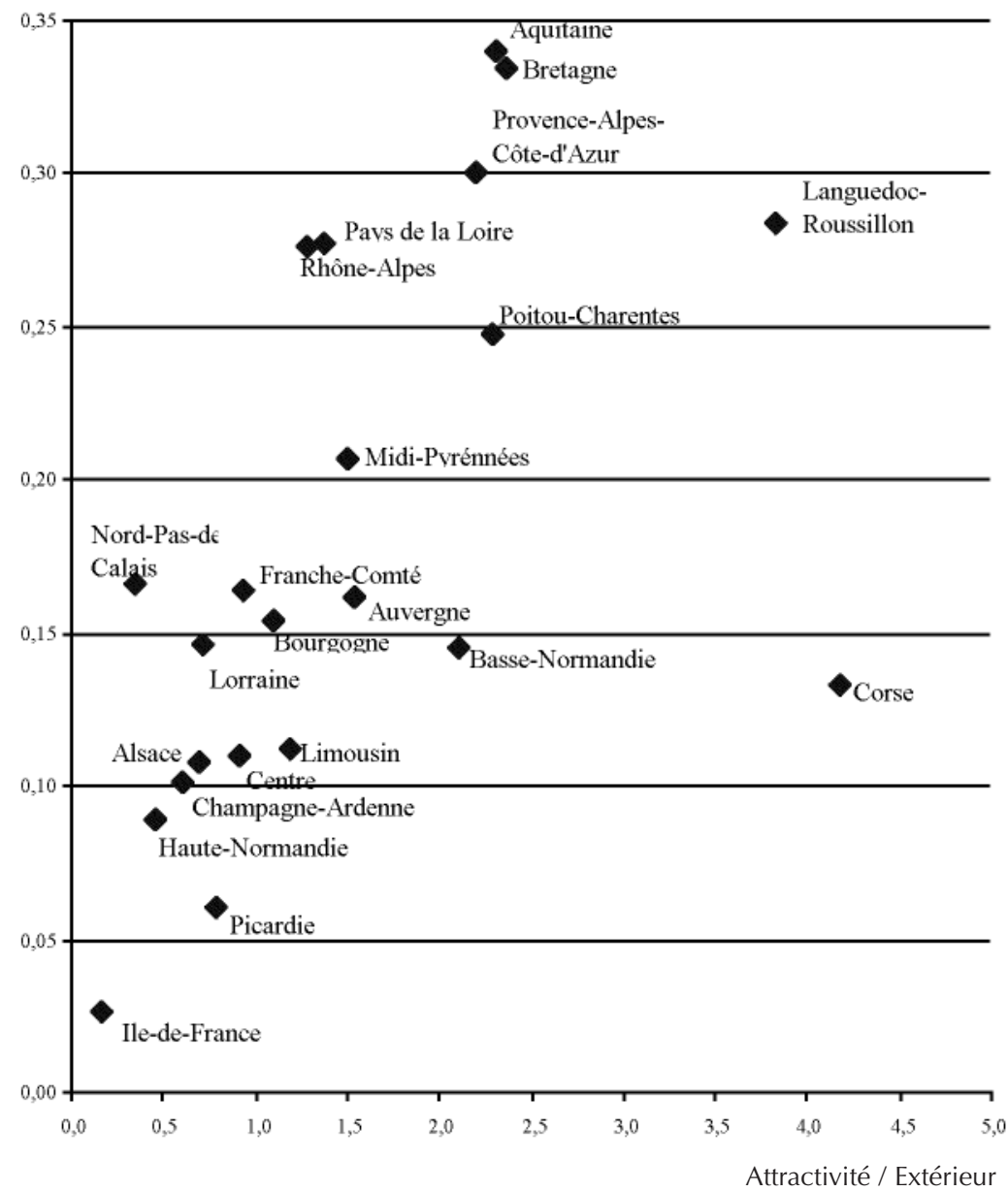

Le coefficient d'attractivité/extérieur est le rapport entre le volume de nuitées touristiques effectuées par les habitants des autres régions françaises dans la région et le volume de nuitées touristiques réalisées par les habitants de la région dans les autres régions françaises. Un coefficient égal à 1 signifie que les flux reçus sont aussi importants que les flux émis.

Le pouvoir casanier représente la proportion de nuitées touristiques effectuées par les habitants de la région dans leur région de résidence.

Source: calcul de l'auteur, SDT 1997-2001-Direction du tourisme/Sofrès région de résidence. Cependant, celles-ci pèsent peu au regard du volume de nuitées en provenance de l'extérieur: les nuitées intrarégionales ne représentent, au final, que $9 \%$ des nuitées touristiques réalisées dans le Languedoc-Roussillon.
La Bretagne, l'Aquitaine et les régions Provence-Alpes-Côte $\mathrm{d}^{\prime}$ Azur et Poitou-Charentes se distinguent, pour leur part, par leur très forte attractivité à l'égard de leurs habitants. L'Aquitaine dispose du pouvoir casanier le plus élevé des régions françaises, 
suivie de près par la Bretagne. Les nuitées effectuées dans la région de résidence représentent, en effet, près de $35 \%$ des nuitées touristiques réalisées par leurs habitants. Elles ont d'ailleurs un poids significatif, puisqu'elles représentent près de $18 \%$ des nuitées touristiques effectuées en Aquitaine et en Bretagne. Ces régions littorales entretiennent également un rapport avantageux avec l'extérieur, puisqu'elles reçoivent deux fois plus de nuitées qu'elles n'en émettent.

Pour les autres régions bénéficiant d'un volume de nuitées par habitant positif, leur attractivité touristique semble diminuer en fonction de leur localisation continentale. Un premier ensemble regroupe les espaces que l'on pourrait qualifier de moyennement attractifs, qui comprennent deux régions littorales (Basse-Normandie, Pays de la Loire) et trois régions de montagne (Rhône-Alpes, Midi-Pyrénées et Auvergne). Elles constituent généralement des destinations secondaires par rapport aux régions du Sud et de l'Ouest citées précédemment. Elles apparaissent moins attractives vis-à-vis de l'extérieur et de leurs propres habitants: les flux émis représentent, ainsi, presque deux tiers des flux touristiques reçus. Seules les régions Pays de la Loire et Rhône-Alpes conservent un pouvoir casanier relativement élevé. Le deuxième ensemble regroupe les espaces peu attractifs à l'égard des flux touristiques. Ceux-ci sont situés dans la partie centrale de la France, il s'agit de la Bourgogne, du Centre, du Limousin et de la Franche-Comté. Ces régions se caractérisent par leur manque d'attractivité vis-à-vis de leurs propres habitants et des habitants des autres régions françaises. Elles ont, en effet, un pouvoir casanier peu développé, et les flux touristiques reçus ne font en fait que compenser les flux touristiques émis.

\section{Le manque d'attractivité des régions du Nord et de I'Est à l'égard de leurs propres habitants et des habitants des autres régions françaises}

À l'autre extrémité du classement se trouvent les régions du Nord et de l'Est, qui peuvent être considérées comme non attractives à l'égard des flux touristiques des Français, le volume de nuitées touristiques émis étant supérieur au volume de nuitées touristiques effectuées dans la région. Elles se caractérisent par un manque d'attractivité à l'égard des habitants des autres régions françaises, qui réalisent peu de séjours touristiques dans ces régions, mais aussi par un manque d'attractivité à l'égard de leurs propres habitants. Le tourisme interrégional semble, en effet, beaucoup moins développé, les habitants de ces régions choisissant leurs principales destinations touristiques à l'exté- rieur de la région de résidence. L'Ile-de-France arrive largement en tête des régions les moins attractives, suivie par la HauteNormandie, le Nord-Pas-de-Calais, la Champagne-Ardenne, la Picardie, l'Alsace et la Lorraine. Mais, là aussi, c'est à chaque fois une combinaison particulière des flux touristiques, qui permet d'expliquer la situation de chaque région, notamment, dans les cas de l'lle-de-France et du Nord-Pas-de-Calais.

L'lle-de-France constitue un cas extrême, puisqu'elle se révèle non seulement très peu attractive vis-à-vis des habitants de la province, mais surtout vis-à-vis de ses propres habitants, ce qui témoigne d'une réelle spécificité par rapport aux autres régions françaises. Le tourisme intrarégional ne représente, en effet, que 2,7\% des nuitées touristiques effectuées par les Franciliens, soit le pourcentage le plus faible des 22 régions métropolitaines. L'Ille-de-France se situe, ainsi, au 14e rang des destinations touristiques des Franciliens alors que, dans la plupart des autres régions, la région de résidence constitue généralement la première ou la seconde destination touristique des habitants. Les Franciliens, qui disposent souvent de revenus élevés et de dessertes de transport de très bonne qualité, quittent donc massivement leur région à l'occasion de leurs déplacements touristiques. Ils ont effectué, ainsi, en moyenne près de 230 millions de nuitées touristiques dans les autres régions françaises par an, ce qui place l'Ile-de-France au premier rang des régions émettrices en terme de nuitées par habitant. Mais l'llede-France apparaît également peu attractive à l'égard des habitants des autres régions françaises, qui choisissent plutôt les régions du Sud et de l'Ouest ou leur région de résidence comme destinations touristiques. Au final, I'lle-de-France entretient le rapport le plus déficitaire avec les autres régions françaises, puisqu'elle émet six fois plus de nuitées touristiques qu'elle n'en reçoit.

Le Nord-Pas-de-Calais, pour sa part, se distingue des autres régions déficitaires par la part relativement importante de son tourisme intrarégional. Celui-ci représente, en effet, 16,6 \% des nuitées touristiques effectuées par les habitants de la région, ce qui est relativement élevé pour les régions non attractives. Mais l'importance du tourisme intrarégional ne doit pas, pour autant, être interprétée comme un reflet de l'attrait particulier exercé par la région sur ses habitants. Le Nord-Pas-de-Calais disposant du plus faible revenu disponible par habitant, on peut, en effet, penser que c'est surtout pour des raisons financières qu'une part importante des nuitées touristiques est réalisée dans la région de résidence. Mais, parallèlement, ces nuitées intrarégionales 
représentent près de 36,6\% des nuitées touristiques effectuées dans la région. Le Nord-Pas-de-Calais est, ainsi, la seule région française dans laquelle le tourisme intrarégional représente un pourcentage aussi élevé et constitue le principal flux des nuitées touristiques réalisées dans la région. Cette situation s'explique en partie par le poids de la population régionale, mais surtout par le manque d'attractivité de la région vis-à-vis des autres régions françaises, ce qui se traduit par de faibles volumes de nuitées touristiques reçus de l'extérieur. Par contre, le Nord-Pasde-Calais étant l'une des régions les plus peuplées, le volume de nuitées touristiques émis est relativement important et la région entretient, par conséquent, un rapport déficitaire avec les autres régions françaises. Elle émet, ainsi, trois fois plus de nuitées touristiques qu'elle n'en reçoit de l'extérieur.

Mais l'attractivité touristique des régions françaises ne peut être évaluée uniquement à partir des flux touristiques des Français en France métropolitaine. II apparaît, en effet, indispensable de prendre en compte également les flux avec l'étranger, qui concernent aussi bien les flux touristiques des Français vers l'étranger que les flux des touristes étrangers en France métropolitaine. Étant donné que la France représente la première destination touristique mondiale et que les régions de destination des touristes étrangers ne sont pas forcément les mêmes que celles des Français, il est possible que les flux touristiques en provenance de l'étranger modifient significativement la géographie de l'attractivité des espaces français à l'égard des mobilités touristiques. En particulier, l'Ile-de-France qui est la région la plus productive de France, mais la moins attractive à l'égard des flux touristiques des Français pourrait voir sa situation basculer en raison de sa position de première destination touristique mondiale.

\section{LA STRUCTURE DES FLUX TOURISTIQUES DES FranÇAIS ET DES ÉTRANGERS EN FranCE MÉTROPOLITAINE}

Avant d'analyser l'attractivité touristique globale des régions françaises à l'égard des flux touristiques, il apparaît nécessaire de présenter, d'abord, les principales tendances qui caractérisent la structure des flux touristiques avec l'étranger.

\section{Les principales caractéristiques des flux touristiques avec l'étranger}

Les déplacements touristiques à l'étranger, même s'ils ont ten- dance à se développer, restent une pratique minoritaire chez les Français. Ainsi, seulement $15 \%$ des nuitées touristiques françaises sont effectuées en moyenne chaque année à l'étranger, principalement dans les pays européens (Espagne, Italie, Grande-Bretagne, Benelux). Les régions françaises participent de manière très inégale à l'émission de ces flux: quatre régions émettent à elles seules plus de la moitié des nuitées touristiques vers l'étranger. II s'agit des régions Ile-de-France, Rhône-Alpes, Provence-Alpes-Côte d'Azur et Aquitaine, qui sont, par ailleurs, les plus peuplées. Au final, les principales régions émettrices de flux touristiques vers l'étranger sont, en terme de nuitées par habitant, I'lle-de-France et les régions Alsace, Rhône-Alpes, Aquitaine et Midi-Pyrénées. On retrouve donc ici les régions frontalières et la région capitale, qui est également la première région émettrice concernant les flux touristiques en France métropolitaine.

Les déplacements touristiques des étrangers représentent, quant à eux, une part essentielle des flux touristiques en France métropolitaine: en moyenne près de $38 \%$ des nuitées effectuées chaque année dans l'hexagone sont réalisées par les étrangers. Mais, à l'exception de l'lle-de-France et de l'Alsace, dans toutes les régions la part des nuitées effectuées par les touristes français est à chaque fois plus élevée que celle des touristes étrangers. Ceux-ci viennent essentiellement des pays européens (Allemagne, Grande-Bretagne, Pays-Bas, Benelux, Italie...) et concentrent leurs séjours sur quelques grandes destinations qui ne correspondent pas toujours à celles des touristes français. Les régions françaises sont, ainsi, inégalement investies par les flux touristiques des étrangers: quatre régions captent à elles seules plus de la moitié des nuitées effectuées par les étrangers en France métropolitaine. Il s'agit de l'lle-deFrance, qui reçoit près de $23 \%$ des nuitées, et des régions du Sud-Est (Provence-Alpes-Côte d'Azur, Rhône-Alpes et Languedoc-Roussillon).

Les principales destinations touristiques des étrangers, en nombre de nuitées par habitant, correspondent aux régions du Sud (Corse, Provence-Alpes-Côte d'Azur, LanguedocRoussillon) et aux régions Ile-de-France, Alsace et Rhône-Alpes. Ainsi, le principal point commun entre la géographie des touristes français et celle des étrangers réside dans l'attrait exercé par les régions méditerranéennes: celles-ci arrivent, dans les deux cas, en tête des destinations touristiques. La principale différence repose sur l'attractivité différenciée de la région lle-deFrance. Elle représente la quatrième destination touristique des 


\section{Carte 2 - L'attractivité des régions françaises à l'égard des flux touristiques des Français et des étrangers}

Source: calcul de l'auteur, SDT 1997-2001

Direction du tourisme/Sofrès

Estimations de la Direction du Tourisme

1997-2001

(C) Arctique
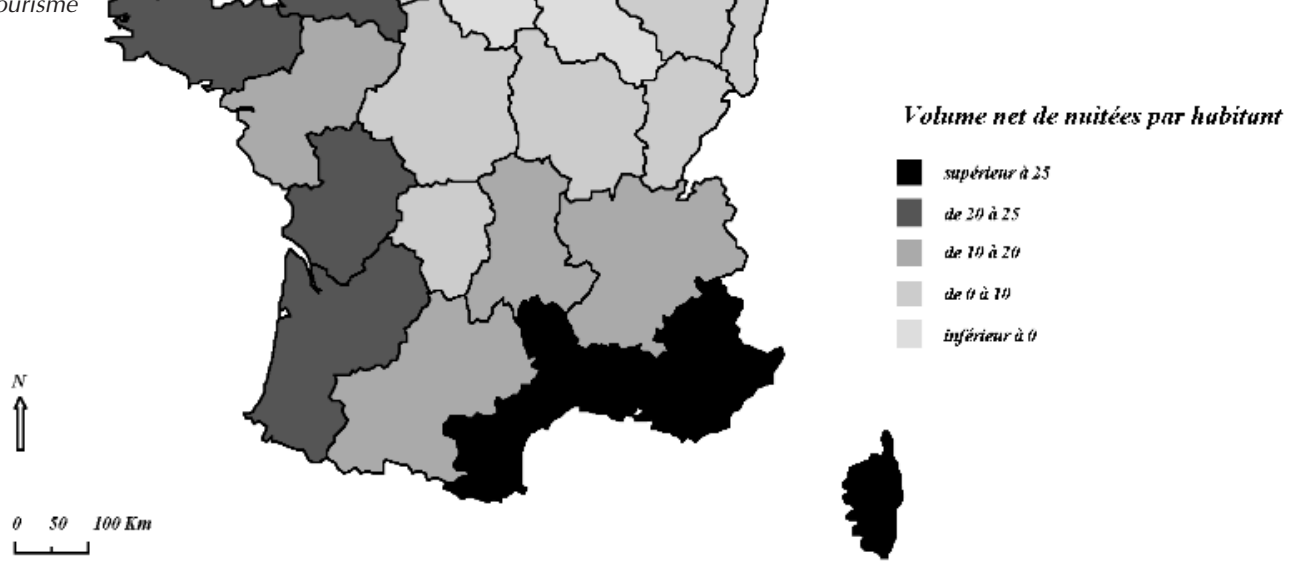

étrangers, Paris jouant ici un rôle clé, mais la dernière destination touristique des Français. Les autres régions très fréquentées par les touristes étrangers concernent des régions frontalières, I'Alsace et Rhône-Alpes, celle-ci étant par ailleurs une destination privilégiée pour les sports d'hiver.

\section{L'attractivité des régions françaises à l'égard des flux touristiques des Français et des étrangers}

La prise en compte des flux avec l'étranger va permettre, à présent, d'évaluer l'attractivité globale des régions françaises à l'égard des flux touristiques. Celle-ci sera mesurée par la différence entre le volume de nuitées touristiques effectuées dans la région et le volume de nuitées touristiques réalisées à l'extérieur de celle-ci. Les flux reçus comprennent dans ce cas les nuitées effectuées dans la région par les touristes étrangers et les touristes français, que ceux-ci soient originaires de la région ou non. Les flux émis correspondent, pour leur part, aux nuitées touristiques effectuées par les habitants de la région dans les autres régions françaises ou à l'étranger. Afin d'évaluer l'importance du volume net de nuitées touristiques effectuées dans chaque région, celui-ci sera rapporté à la population régionale.

La prise en compte des flux avec l'étranger modifie en fait peu l'attractivité des régions françaises à l'égard des flux touristiques. Il existe toujours une césure nette entre les régions du Sud et de l'Ouest, qui sont très attractives vis-à-vis des touristes français et étrangers, et les régions du Nord et de l'Est, qui ne le sont pas ou peu. Les déplacements touristiques des Français à l'étranger influent à la marge, car ils engendrent des volumes de nuitées touristiques beaucoup moins importants que les déplacements des touristes étrangers en France métropolitaine. Ce sont donc essentiellement les flux touristiques en provenance de l'étranger, qui vont jouer un rôle déterminant dans l'évolution de la situation des régions françaises.

Au final, les régions françaises les plus attractives à l'égard des flux touristiques correspondent aux régions littorales du Sud et de l'Ouest. L'attractivité déjà très forte de ces espaces vis-àvis des touristes français est, en effet, renforcée par leur attractivité à l'égard des étrangers. C'est le cas, notamment, des régions méditerranéennes, qui sont très plébiscitées par les touristes étrangers et qui arrivent donc largement en tête du classement avec, par ordre croissant, la Corse, le Languedoc-Roussillon et la région Provence-Alpes-Côte d'Azur. Les régions de la façade atlantique sont également très attractives à l'égard des flux touristiques: l'Aquitaine, la Bretagne, la région Poitou-Charentes et la Basse-Normandie. Mais, contrairement à celles du Sud, elles semblent davantage attirer les touristes français que les touristes étrangers.

Les régions moyennement, voire peu attractives à l'égard des flux touristiques sont situées dans les parties centrales et septentrionales de l'hexagone. Pour certaines, la prise en compte des déplacements touristiques avec l'étranger a permis de 


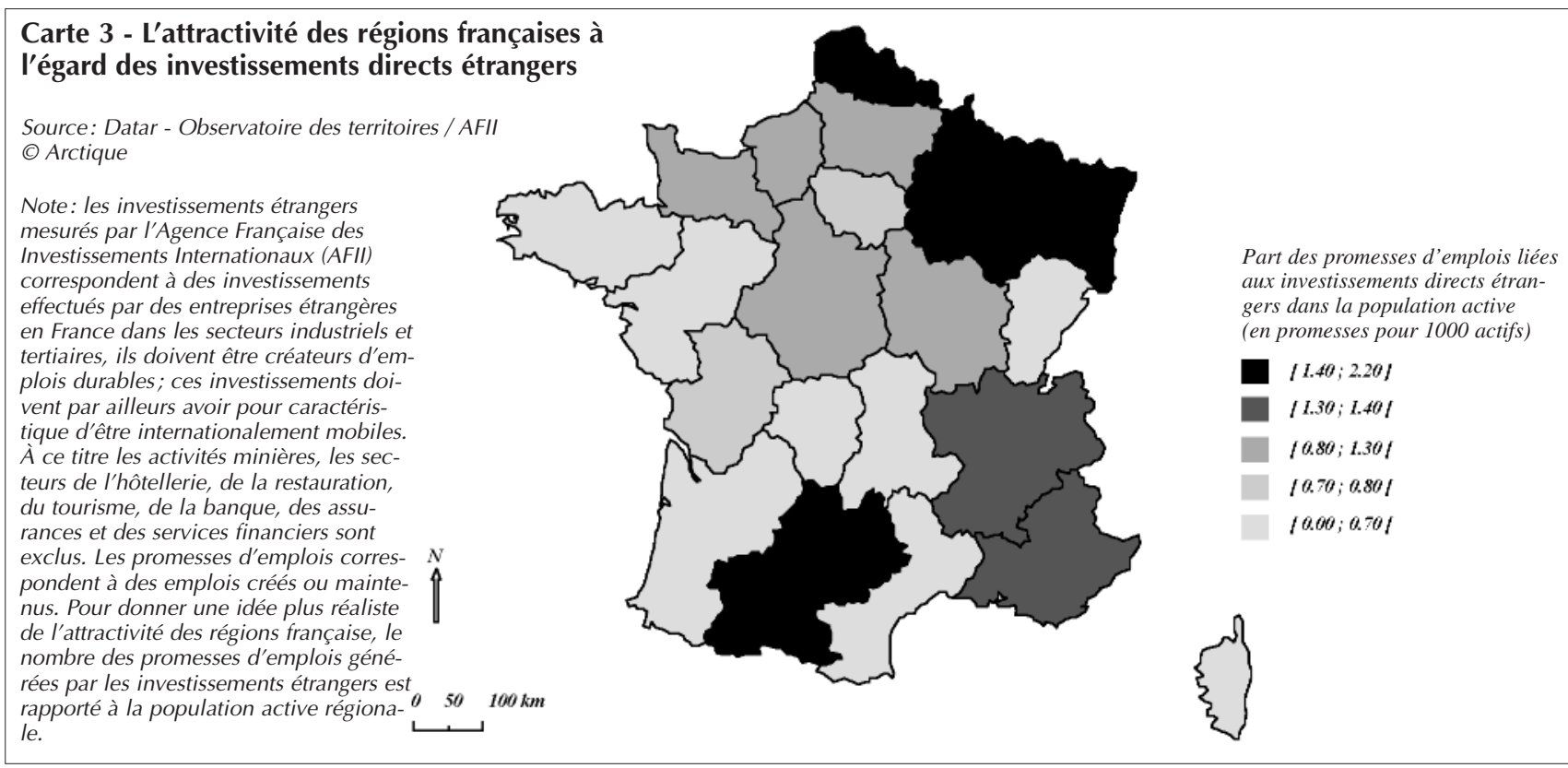

passer d'une situation déficitaire à une situation bénéficiaire. Ainsi, seules quatre régions apparaissent déficitaires au lieu de sept auparavant, les nuitées touristiques des étrangers venant compenser les nuitées effectuées par les habitants de ces régions à l'extérieur, en France ou à l'étranger. Certaines régions du Nord-Est bénéficient, ainsi, désormais d'un volume net de nuitées touristiques par habitant positif: c'est le cas de I'Alsace, de la Lorraine et de la Picardie. La forte attractivité de l'Alsace vis-à-vis des touristes étrangers lui permet de compenser son manque d'attractivité vis-à-vis des touristes français, et la région qui était auparavant dans une situation déficitaire se trouve finalement dans une position intermédiaire. La Lorraine et la Picardie restent, pour leur part, peu attractives aussi bien à l'égard des flux touristiques des Français que des étrangers, les nuitées touristiques étrangères apparaissant juste suffisantes pour équilibrer le rapport déficitaire que les régions entretenaient avec l'extérieur.

Enfin, les régions non attractives à l'égard des flux touristiques correspondent toujours aux régions du Nord et de l'Est. Les flux touristiques avec l'étranger ne permettent pas, dans leur cas, de modifier leur situation déficitaire initiale. Pour la HauteNormandie, le Nord-Pas-de-Calais et la Champagne-Ardenne, le manque d'attractivité à l'égard des touristes français semble se doubler d'un manque d'attractivité à l'égard des touristes étrangers. La proximité de ces régions avec les pays d'origine des touristes étrangers, qui aurait pu constituer un avantage, ne semble pas avoir d'effet sur leur fréquentation. Ainsi, dans ces régions les flux touristiques reçus ne permettent pas de compenser les flux touristiques émis. L'lle-de-France se trouve, pour sa part, dans une situation très différente, puisqu'elle se distingue par sa très forte attractivité à l'égard des touristes étrangers. Elle constitue, en effet, la première destination touristique mondiale et l'une des principales destinations des étrangers qui visitent la France. Elle capte, en effet, à elle seule près du quart des nuitées touristiques étrangères effectuées en France métropolitaine, ce qui la place au cinquième rang des régions réceptrices en terme de nuitées par habitant. Cependant, la très forte attractivité de l'Ile-de-France à l'égard des touristes étrangers n'arrive pas à compenser son manque d'attractivité à l'égard des touristes français. Or, celui-ci s'avère déterminant: non seulement l'lle-de-France ne constitue pas une destination très prisée des provinciaux, mais en plus elle se révèle très peu attractive pour ses propres habitants (6). Par conséquent, les déplacements touristiques particulièrement nombreux des Franciliens profitent essentiellement aux autres régions françaises et à l'étranger. Ainsi, bien que l'image très répandue de l'lle-deFrance comme première destination touristique mondiale soit avérée, la prise en compte des déplacements des touristes français et notamment des Franciliens contribue à mettre en évidence le caractère finalement peu attractif de la région à l'égard des flux touristiques.

Au final, I'analyse de la structure des flux touristiques en 
France métropolitaine met en évidence l'attractivité très différenciée des régions à l'égard des touristes français et étrangers. Les régions littorales du Sud et de l'Ouest se révèlent, ainsi, fortement attractives non seulement à l'égard de leurs habitants, mais également à l'égard des touristes originaires des autres régions françaises et de l'étranger. Les régions situées au sud de l'lle-de-France et ne bénéficiant pas d'un accès la mer se situent, pour leur part, dans une situation intermédiaire. Les régions du Nord et de l'Est, enfin, se caractérisent par leur manque d'attractivité aussi bien à l'égard de leurs propres habitants que des touristes extérieurs à la région, ce qui se traduit par un solde déficitaire, les nuitées effectuées à l'extérieur de la région étant supérieures aux nuitées réalisées dans la région. Cette analyse de l'attractivité des espaces français à l'égard des flux touristiques contribue, ainsi, à souligner une autre dimension de l'attractivité territoriale en dévoilant une nouvelle géographie des espaces attractifs. En effet, dans un contexte de mobilité croissante des activités et de concurrence accrue entre espaces, I'attractivité des territoires est habituellement définie par leur capacité à attirer les entreprises et elle est généralement évaluée à partir d'indicateurs spécifiques comme les investissements internationaux effectués localement. Or, la comparaison de la géographie de l'attractivité des espaces français à l'égard des flux d'investissements internationaux (7) et des flux touristiques révèle une France inverse: les régions du Nord et de l'Est apparaissent dans leur ensemble très attractives vis-à-vis des premiers et peu vis-à-vis des seconds, les régions de l'Ouest et du Sud, pour leur part, connaissent généralement la situation inverse. Ces éléments montrent, à travers l'exemple des mobilités touristiques, que les espaces attractifs à l'égard des entreprises ne le sont pas systématiquement à l'égard des individus, et que, par conséquent, les espaces de la production ne correspondent pas toujours à ceux de la consommation. Ainsi, l'Illede-France qui est aujourd'hui la principale région bénéficiaire de ce phénomène de concentration des activités et qui est au premier rang des régions les plus productives, est également la région qui possède de loin le solde touristique le plus déficitai- re de toutes les régions françaises, et ce malgré son attractivité vis-à-vis des touristes étrangers. Or, les déplacements touristiques des Franciliens se traduisent sur le plan économique par un déplacement de consommation du lieu de résidence vers le lieu de séjour, ce qui pourrait avoir des conséquences pour les économies locales. En effet, à travers la très forte mobilité touristique des Franciliens, une partie de la richesse produite localement ne bénéficie pas au territoire de résidence mais au territoire de destination. Ces déplacements de consommation semblent, ainsi, profiter essentiellement aux régions de l'Ouest et du Sud, qui sont, par ailleurs, moins favorisées sur le plan productif. Si, dans le cas de l'lle-de-France, ces déplacements de consommation semblent plutôt favorables à l'aménagement du territoire, puisqu'ils s'apparentent à un transfert de richesse de la région la plus riche de France vers les régions périphériques de l'Ouest et du Sud, ils pourraient avoir des effets moins vertueux dans d'autres espaces. Ainsi, le manque d'attractivité touristique du Nord-Pas-de-Calais, de la Picardie et de la Lorraine, qui se traduit par l'émission de flux importants hors de la région, pourrait avoir des effets préjudiciables pour ces espaces qui connaissent par ailleurs des difficultés économiques importantes. En conclusion, cette approche, qui nécessiterait évidemment d'être approfondie, vise à montrer que l'analyse des économies locales et de l'attractivité territoriale devrait être envisagée dans une double dimension. Si la mobilité des entreprises contribue aujourd'hui incontestablement à redessiner la géographie économique de la production, la mobilité croissante des individus, favorisée par l'augmentation du temps libre, semble esquisser également une géographie économique de la consommation qui mériterait d'être prise en compte de manière complémentaire.

Patricia Lejoux est ATER à I'UFR de Géographie et d'Aménagement de I'Université Lille I et doctorante au Centre de Recherche sur l'Espace, les Transports, I'Environnement et les Institutions Locales

(C.R.E.T.E.I.L.), à I'Institut d'Urbanisme de Paris, Université Paris XII. Elle prépare un doctorat en aménagement sous la direction de Laurent Davezies, qui porte sur les thématiques du développement local et des mobilités touristiques.

Courriel: lejoux@univ-paris12.fr

\section{BibliographIE}

BACCAINI B., 2001, "Les migrations en France entre 1990 et 1999, les régions de l'Ouest de plus en plus attractives », Insee Première, $\mathrm{n}^{\circ} 758$, février, $4 \mathrm{p}$.

Bonnin P., De Villanova R. (dirs.), 1999, D'une maison l'autre: parcours et mobilités résidentielles, Grane, Ed. Créaphis, $366 \mathrm{p}$.

Claval P., 2003, La géographie du XXle siècle, Paris,
L'Harmattan, $243 \mathrm{p}$.

DAveZies L., 2004, « Développement local: le déménagement des Français », Futuribles, n²95, mars, pp. 43-56.

DAveZIES L., 2003, " Les inégalités territoriales, la lutte du pot de terre contre le pot de fer? ", in L'aménagement durable: défis et politiques, sous la direction de Wachter S., La Tour d'Aigues, Éditions de l'Aube, 195 p. 
Dubost F. (dir.), 1998, L'autre maison: la « résidence secondaire ", refuge des générations, Paris, Éditions Autrement, $183 \mathrm{p}$.

Gerard-Varet L.-A., Mougeot M., 2001, « L'État et l'aménagement du territoire » in L'Aménagement du territoire, sous la direction de Guigou J.-L. et alii, rapport du Conseil d'Analyse Économique, Paris, La Documentation française, pp. 44-108.

INSEE, 2005, Le tourisme en France, Paris: INSEE, coll. INSEERéférences, $173 \mathrm{p}$.

KNAFOU R., 2000, " Les mobilités touristiques et de loisirs et le système global des mobilités » in Les territoires de la mobilité, sous la direction de Bonnet M., Desjeux D., Paris, PUF, pp. 85-94.

KRUGMAN P., 1991, "Increasing Returns and Economic Geography », Journal of Political Economy, n99, pp.483499.
Lejoux P., 2003, "La géographie des flux de dépenses touristiques des Français en France », La lettre de I'observation du tourisme, $\mathrm{n}^{\circ} 74$, Paris, ONT, octobre.

Lejoux P., 2002, Esquisse d'une géographie des flux de revenu/consommation associés aux pratiques touristiques, recherche pour le Ministère du Tourisme/Direction du Tourisme, L'OEIL, Université Paris XII.

Terrier C., Khiati A., Sylvander M., 2005, « En haute saison touristique, la population double dans certains départements », Insee Première, $\mathrm{n}^{\circ} 1050,4 \mathrm{p}$.

Thisse J.-F., VAN Ypersele T., 1999, " Métropoles et concurrence territoriale », Économie et Statistique, n³26-327, septembre, pp. 19-30.

Veltz P., 1996, Mondialisation, villes et territoires: l'économie d'archipel, Paris, PUF, 264 p.

VIARD J., 1994, La société d'archipel où les territoires du village global, La Tour d'Aigues, Éditions de l'Aube, 128 p.

\section{NOTES}

(1) Voir en particulier le dossier thématique « Des territoires plus attractifs » dans le numéro 61 de la revue Pouvoirs Locaux, paru en juin 2004.

(2) Voir notamment les différents manuels publiés au cours de ces dernières années:

Dinéty J.-C., Proust E., 2002, Géographie du tourisme, Paris, BPI, 158 p.

Dewailly J.-M., Flament E., 2000, Le tourisme, Paris, SEDES, 191 p.

Lozato-Giotart J.-P., 2003, Géographie du tourisme: de l'espace consommé à l'espace maîtrisé, Paris, Pearson Éducation France, 330 p.

Stock M. (coor.), 2003, Le tourisme: acteurs, lieux et enjeux, Paris, Belin, 299 p.

(3) Cette place de première destination touristique mondiale doit cependant être relativisée. S'il est indéniable que les attraits touristiques de la France expliquent en grande partie l'importance du volume d'arrivées de touristes internationaux, sa situation géographique au cœur de l'Europe joue également un rôle essentiel. En effet, la France constitue un point de passage obligé entre les pays émetteurs du Nord et les pays récepteurs du Sud, ainsi un touriste hollandais qui se rend en Espagne et qui passe au moins une nuitée en France pour faire une étape sera comptabilisé comme une arrivée d'un touriste international en France alors que le touriste ne fait en réalité que traverser le pays. Ce phénomène est loin d'être négligeable puisqu'en 2002 plus de $21 \%$ des séjours des touristes internationaux étaient d'une durée d'une nuitée seulement.

(4) Voir notamment:
Potier F., Terrier C., Sylvander M., Zegel P., à paraître, Atlas des mobilités touristiques, Paris, Éditions Autrement.

Potier F., 2002, "La République du temps libre n'est pas une et indivisible » in La France des temps libres et des vacances, sous la direction de Viard J., Potier F., Urbain J.D., La Tour d'Aigues, Éditions de l'Aube, pp. 149-163.

(5) Voir notamment:

Direction du tourisme, 2005, Mémento du tourisme, Paris, ONT, $135 \mathrm{p}$.

Observatoire National du Tourisme, 2003, Atlas du tourisme, Paris, ONT, 112 p.

(6) L'importance des déplacements extra-régionaux semble constituer une caractéristique forte de l'Ile-de-France, puisque même dans le cadre de l'excursionnisme, c'est-à-dire des déplacements à la journée, ce sont les Franciliens qui se déplacent le plus en dehors de leur région: 96 \% de leurs déplacements à la journée sont ainsi effectués à l'extérieur de la région de résidence. Mais une partie de ces déplacements est liée, ici, à des déplacements domicile-travail effectués à plus de 100 km du domicile (INSEE, 2005).

(7) Les investissements étrangers mesurés par l'Agence Française des Investissements Internationaux (AFII) correspondent à des investissements effectués par des entreprises étrangères en France dans les secteurs industriels et tertiaires, qui doivent être créateurs d'emplois durables. Ces investissements doivent par ailleurs avoir pour caractéristique d'être internationalement mobiles. À ce titre les activités minières, les secteurs de l'hôtellerie, de la restauration, du tourisme, de la banque, des assurances et des services financiers sont exclus. 